\title{
Predicting size effects in nickel-base single crystal superalloys with the Discrete-Continuous Model
}

\author{
Aurélien Vattré* — Benoit Devincre** — Arjen Roos* \\ Frédéric Feyel*
}

*DMSM (ONERA)

29 Avenue de la Division Leclerc, F-92322 Châtillon cedex

\{Aurelien.Vattre,Arjen.Roos, Frederic.Feyel\}@onera.fr

** LEM (CNRS-ONERA)

29 Avenue de la Division Leclerc, F-92322 Châtillon cedex

Benoit.Devincre@onera.fr

\begin{abstract}
The Discrete-Continuous Model, a coupling between dislocation dynamics and finite elements simulations, is used for modelling size effects in the mechanical properties of single-crystal superalloys. Both formation and evolution of the dislocation microstructures are analysed, and the crucial role of the storage of signed dislocations at the interfaces is discussed. The onset of plasticity is found to scale as the inverse of the channel width, and polarised dislocation networks at the interfaces significantly increase the flow stress with respect to a bulk crystal.

RÉSUMÉ. Le Modèle Discret-Continu, utilisant un couplage entre simulations de dynamique des dislocations et des éléments finis, permet un calcul de l'effet de taille contrôlant le comportement mécanique d'un superalliage monocristallin. La formation et l'évolution des microstructures de dislocations sont étudiées, et le rôle majeur de l'accumulation des dislocations signées aux interfaces est discuté. L'écoulement plastique est initialement controlé par la largeur des couloirs de matrice et un éccrouissage fort est justifié par une accumulation de dislocations d'interfaces polarisées.

KEYWORDS: single-crystal superalloys, size effects, coupling dislocations dynamics-finite elements.

MOTS-CLÉS : superalliages monocristallins, effets de taille, couplage dynamique des dislocations-éléments finis.
\end{abstract}




\section{Introduction}

Single-crystal nickel-base $\gamma / \gamma^{\prime}$ superalloys are extensively used in applications requiring high strength and fatigue resistance up to elevated temperatures. They mainly consist of two phases: a ductile disordered fcc Ni matrix (the $\gamma$ phase) and hard coherent $\mathrm{L}_{2}$ ordered precipitates (the $\gamma^{\prime}$ phase), regularly distributed in roughly cuboidal shapes with faces parallel to the $\{100\}$ planes. A substantial increase of the macroscopic flow stress of the single crystal can be achieved by decreasing the precipitate size while keeping the volume fraction constant, for instance as measured by Duhl (Duhl, 1987) for PWA1480, a representative single-crystal superalloy.

One approach to model their mechanical response is by using a periodic unit cell representation of the microstructure, where the two phases are represented as a parallelepiped with the mechanical properties of the precipitate, embedded inside another with those of the matrix. Many authors have modelled the mechanical behaviour for an idealised microstructure using the Finite Element (FE) method, for instance (Pollock et al., 1992; Nouailhas et al., 1997). However, the effect of the precipitate size cannot be predicted by classical continuum theories, because they do not contain an intrinsic length scale. Several more sophisticated theories have been developed which, in various ways, include a length-scale dependence (Acharya et al., 2000; Gurtin, 2000). Some attempt to incorporate an internal length through the concept of Geometrically Necessary Dislocations (GND) introduced by Nye (Nye, 1953). Non-local continuum plasticity theories such as (Forest et al., 2000) or strain-gradient based plasticity using the evolution of GND densities have also been applied to the simulation of the flow behaviour of single crystal superalloys (Busso et al., 2000).

Nevertheless, Dislocation Dynamics (DD) simulation appears to be the most appropriate tool to address such microstructural issues and the origin of the size effect. DD simulations have already been carried out on a composite material subjected to simple shear by (Cleveringa et al., 1997; Cleveringa et al., 1998) or on a constrained strip (Shu et al., 2001). Both studies were carried out in 2D, and the main feature controlling the plastic response is the formation of pile-ups. However, for the single crystal $\gamma / \gamma^{\prime}$ superalloys, such pile-ups are not observed experimentally so the size effects observed there must be caused by another mechanism. This is why full 3D simulations are needed here, as in (Groh et al., 2005).

In this article, the effect of the channel width at constant $\gamma^{\prime}$ volume fraction on the tensile plastic deformation of single-crystal $\gamma / \gamma^{\prime}$ superalloys is investigated using the Discrete-Continuous Model (DCM). Emphasis is put on the elementary mechanisms governing the yield stress in multislip conditions.

\section{The Discrete-Continuous Model}

The DCM (Lemarchand et al., 2001) is a method to solve complex boundary value problems of dislocated bodies, which cannot be done by conventional DD codes. Competing methods exist in the literature, for instance (Van der Giessen et 
al., 1995; Fivel et al., 1999; Zbib et al., 2002), but it is beyond the scope of this article to discuss their relative merits and drawbacks. The DCM consists of two codes, microMégas (DD) and ZéBuLoN (FE), coupled into a unified system. The DD code accounts for the dislocation dynamics and computes the plastic strain increments generated by dislocation glide and the associated stress, just as any other classical constitutive law. The FE code predicts the strain increment and verifies the mechanical equilibrium of the boundary value problem, using the plastic strain distribution and the stresses given by the DD simulation.

In this approach, material defects are modeled using eigenstrains (Mura, 1987). In the framework of elastic theory, dislocation loops can be described as a set of thin plate-like coherent inclusions (Figures $1 \mathrm{a}-\mathrm{c}$ ). This elementary dislocation slip, whose direction and amplitude is given by the Burgers vector $\mathbf{b}$, is physically localised on one cristallographic slip plane and bounded by a singularity at the dislocation line. In the DCM scheme, the displacement jump associated to the dislocation loop propagates with the dislocation line on a specific crystallographic plane. It is then distributed over a slab of thickness $h$. Outside the sheared volume, such distributions reproduce the elastic fields of dislocations well (Figure 1d).

The simulated cell contains a precipitate surrounded by six channels, and is discretised by 4096 quadratic FE and 56361 degrees of freedom. Periodic Boundary Conditions (PBC) are applied at all external boundaries. Calculations were performed on three different unit cells scaled up with the same mesh discretisation. Two cases are shown in Figure 2. In all simulations, the $\gamma^{\prime}$ volume fraction was 0.61, yielding a constant ratio between channel width and precipitate size of 0.16 , while varying the channel width $w=0.08,0.16$, and $0.24 \mu \mathrm{m}$. In order to avoid PBC artifacts due to

a.

b.

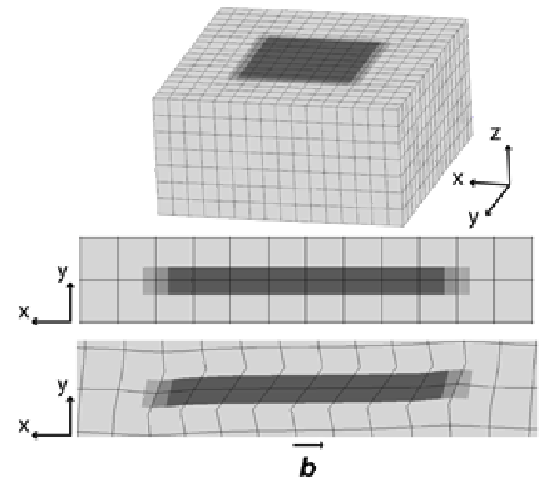

d.

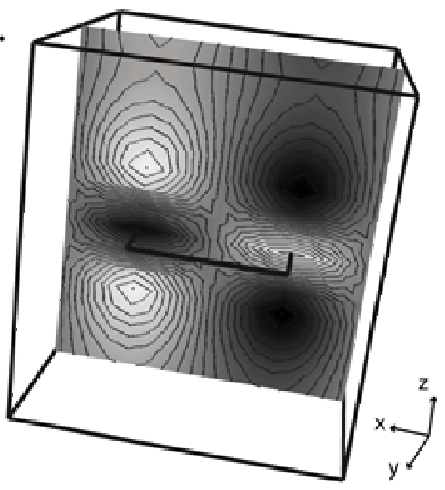

Figure 1. (a) Eigenstrain $\varepsilon_{31}^{p}$ associated to a dipolar dislocation loop at the integration points in a finite element mesh. (b) View in $2 D$. (c) The presence of the eigenstrain creates a displacement jump of value $b(\times 200)$. (d) Stress field $\sigma_{31}$ due to the loop, calculated by the DCM 
a.

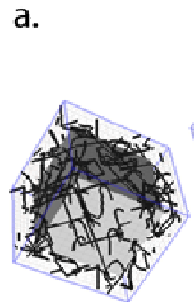

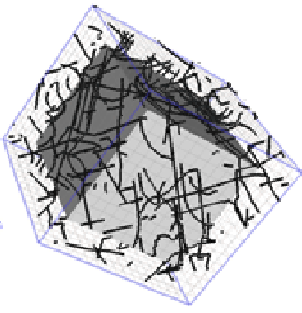

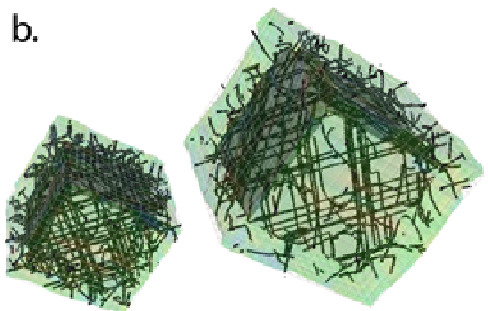

Figure 2. Two simulated volumes with different channel widths and precipitate sizes but with the same precipitate volume fraction of 0.61. Dislocation microstructures (a) before any mechanical loading and (b) at $0.2 \%$ plastic strain

self-annihilation of dislocation loops (Madec et al., 2003), the precipitates have orthorhombic dimensions $0.48 x \times 0.50 x \times 0.52 x \mu \mathrm{m}^{3}$, with $x$ a scaling parameter to assess the effect of precipitate size with $x=1,2$ or 3 .

Isotropic elasticity is assumed everywhere with shear modulus $\mu=51 \mathrm{GPa}$ and Poisson ratio $\nu=0.37$. In the DD code, the lattice parameter $a=0.36 \mathrm{~nm}$ is assumed identical in both phases. This implies a Burgers vector of length $b \approx 0.25 \mathrm{~nm}$.

Shearing of the precipitates has been incorporated into the DCM (Vattré et al., 2009) and it correctly reproduces the anomalous temperature dependence (Westbrook, 1967) of the bowing-assisted cutting process. In the $\gamma^{\prime}$ phase the main parameter controlling the dynamics of precipitate shearing is a configuration stress $\tau_{\mathrm{APB}}$, which accounts for an anti-phase boundary (APB) creation or recovery through the APB energy $\gamma^{\mathrm{APB}}$. All simulations in this paper are carried out at $850^{\circ} \mathrm{C}$ with $\gamma^{\mathrm{APB}}=350 \mathrm{~mJ} . \mathrm{m}^{-2}$ (Veyssière et al., 1997). At this temperature dislocations do not penetrate into the precipitates, which is consistent with experimental observations at small plastic deformations (Carry et al., 1977; Pollock et al., 1992), where the dislocation loops move through the channels, bowing out between the precipitates when a threshold stress $\tau^{\text {Orowan }} \approx \mu b / w$ is reached.

Initial conditions in DCM simulations should be as close as possible to the experimental conditions in terms of dislocation density, dislocation source distribution and dislocation entanglement. The initial configuration in a DCM simulation is generated with a Volterra shearing procedure (Devincre et al., 2003). This procedure is needed to set up an eigenstrain distribution in the FE mesh that is mechanically compatible with the initial dislocation microstructure. The initial dislocation distribution consists only of dipolar loops, i.e. four connected edge segments with the same Burgers vector (Figure 1). In this manner, dislocation segments act as Frank-Read sources and build up an interconnected dislocation network free of pending lines. A total dislocation density is homogeneously assigned to the 12 octahedral slip systems in the form of 24 dipolar loops. 
Table 1. Parameters used in the DCM simulations

\begin{tabular}{|c|c|c|c|}
\hline Parameter & Symbol & Value & Unit \\
\hline \hline Magnitude of Burgers vector & $b$ & $2.5 \times 10^{-10}$ & $\mathrm{~m}$ \\
Shear modulus & $\mu$ & 51 & $\mathrm{GPa}$ \\
Poisson ratio & $\nu$ & 0.37 & - \\
Damping constant & $B$ & $1 \times 10^{-4}$ & $\mathrm{~Pa} . \mathrm{s}$ \\
Lattice friction $\gamma$ phase & $\tau_{F}$ & 107 & $\mathrm{MPa}$ \\
APB energy & $\gamma^{\mathrm{APB}}$ & 350 & $\mathrm{~mJ}^{-2}$ \\
Initial dislocation density & $\rho_{0}$ & $6.2 \times 10^{13}$ & $\mathrm{~m}^{-2}$ \\
Misfit strain & $\delta$ & -3 & $\%$ \\
Imposed strain rate & $\dot{\gamma}$ & 20 & $\mathrm{~s}^{-1}$ \\
\hline
\end{tabular}

In real materials, an additional strengthening mechanism is due to the lattice mismatch between the two phases. In the DCM simulations, it can be accounted for with a preliminary thermo-elastic FE calculation, in which the microstructure is artificially heated from an initial state without misfit at room temperature. This requires two thermal expansion coefficients, $a^{\gamma}$ and $a^{\gamma^{\prime}}$, respectively associated to the $\gamma$ matrix and $\gamma^{\prime}$ precipitate, and the appropriate heating interval $\Delta T$. The misfit strain $\delta$ is then given by $\left(a^{\gamma^{\prime}}-a^{\gamma}\right) \Delta T$, here equal to $-3 \%$ at $850^{\circ} \mathrm{C}$.

As explained in (Vattré et al., 2010), an initial relaxation, where the APB energy and the coherency stress drive the dislocations towards the interfaces, is needed. The dislocation configuration is supposed to be relaxed when there are no more dislocations inside the precipitate. After the process, the total dislocation density on the 12 octahedral slip systems is $6.2 \times 10^{13} \mathrm{~m}^{-2}$. As shown in Figure $2 \mathrm{a}$, the dislocations have moved to the interfaces where they locally reduce the coherency stress.

During the simulations, the relaxed dislocation configurations for the three microstructures are subjected to a pure tensile loading along the [001] crystallographic axis. In order to run calculations within a reasonable time, a high resolved strain rate of $\dot{\gamma}=20 \mathrm{~s}^{-1}$ is imposed. By comparison with the relaxed dislocation network in Figure $2 a$, Figure $2 b$ shows the dense interfacial dislocation network obtained at $0.2 \%$ plastic strain. In these calculations, the DD simulation time step is $5 \times 10^{-11} \mathrm{~s}$ and the FE time step is ten times as long. All simulation parameters are listed in Table (1).

\section{Simulation results}

\subsection{Mechanical responses}

Figure $3 \mathrm{a}$ shows the simulated stress-strain curves. Two different stages can be distinguished. A first stage corresponds to a transient regime from zero to $0.01 \%$ plastic strain, during which plastic deformation mainly results from the bowout of the 


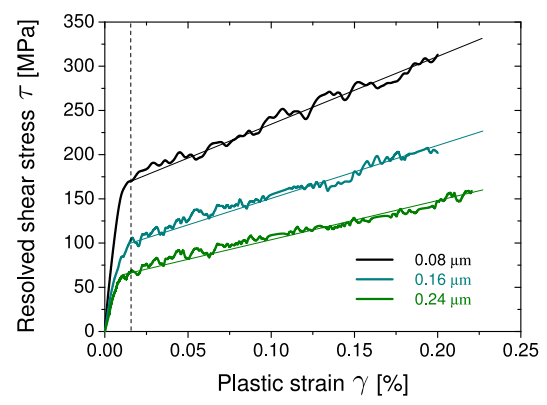

(a)

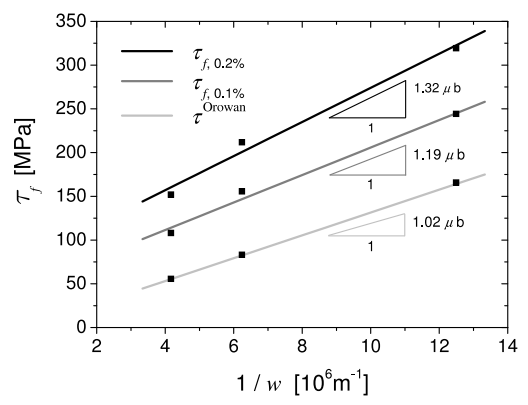

(b)

Figure 3. (a) Effect of morphologies on the simulated stress-strain curves. The solid straight lines represent linear fits, and the dotted vertical line indicates the boundary between the first and second stages. (b) Flow stress $\tau_{f}$ at $0.01 \%, 0.1 \%$ and $0.2 \%$ plastic strain versus the inverse of the width $w$ of the channel. The straight lines represent fits of the dependence on $1 / w$

dislocations initially present inside the channels. A second stage corresponds to the irreversible plastic deformation from $0.01 \%$ plastic strain up to the end of simulations. During this second stage, the strain hardening rate is constant and plastic deformation arises from dislocations passing around the precipitates. Irrespective of the strain value, the flow stress increases significantly with decreasing channel width.

\subsection{Flow stress and work-hardening}

To quantify the size effect, the values of the $0.01 \%$ yield stress (called Orowan stress here) $\tau^{\text {Orowan }}$, the values of the flow stresses at $0.1 \%$ and $0.2 \%$ plastic strain, $\tau_{f, 0.1 \%}$ and $\tau_{f, 0.2 \%}$ respectively, and the rate of hardening $\theta$ are given in Table (2). The instantaneous work-hardening rate is defined as $\theta=\Delta \tau / \Delta \gamma$, with $\tau$ and $\gamma$ the shear strain and the flow stress resolved on the most active octahedral slip system.

Table 2. Flow stress and hardening of the three morphologies of a $\gamma / \gamma^{\prime}$ single crystal superalloy. The symbols $\tau^{\text {Orowan }}, \tau_{f, 0.1 \%}, \tau_{f, 0.2 \%}$ and $\theta$ denote the Orowan yield stress at $0.01 \%$ plastic strain, the flow stresses at $0.1 \%$ and $0.2 \%$ plastic strain and the hardening rate, respectively

\begin{tabular}{|c|c|c|c|c|c|}
\hline$x$ & $w(\mu m)$ & $\tau^{\text {Orowan }}(\mathrm{MPa})$ & $\tau_{f, 0.1 \%}(\mathrm{MPa})$ & $\tau_{f, 0.2 \%}(\mathrm{MPa})$ & $\theta / \mu$ \\
\hline \hline 1 & 0.08 & 164 & 243 & 320 & 0.149 \\
\hline 2 & 0.16 & 83 & 155 & 212 & 0.142 \\
\hline 3 & 0.24 & 55 & 108 & 152 & 0.134 \\
\hline
\end{tabular}


The flow stress $\tau_{f}$ increases with decreasing specimen size. To quantitatively assess this size effect, the flow stress is plotted in Figure $3 \mathrm{~b}$ at $0.01 \%, 0.1 \%$ and $0.2 \%$ plastic strain as a function of $1 / w$, showing that the simulation results can be related to a $1 / w$ scaling law. In particular, the value of the $0.01 \%$ yield strength corresponds quantitatively to the critical macroscopic Orowan stress, expressed here as

$$
\tau^{\text {Orowan }}=1.02 \mu b / w .
$$

In addition, Figure $3 \mathrm{~b}$ depicts the values of the flow stress $\tau_{f}$ at $0.1 \%$ and $0.2 \%$ plastic strain, as a function of $1 / w$. The Orowan-like law fits well to the data in Figure $3 \mathrm{~b}$ at all plastic strain levels and the flow stress may be given as

$$
\tau_{f}=k \mu b / w,
$$

with $k=1.19$ at $0.1 \%$ plastic strain and $k=1.32$ at $0.2 \%$ plastic strain. Equation (2) means that the flow stress is strongly dependent on the channel width.

The effect of specimen size on hardening is quantified in Table (2) by the values of $\theta / \mu$. The work-hardening rate $\theta$ increases with decreasing specimen size. These values can be related to dislocation storage at the interfaces, i.e. the size dependence of $\theta$ is related to the dislocation density deposited there, as will be discussed next.

\subsection{Dislocation densities}

Figure 4 a shows the evolution of the dislocation density $\rho$ with plastic strain. At the same $\gamma^{\prime}$ volume fraction, the dislocation density increases linearly with strain, more rapidly so for smaller channel widths. Figure $4 \mathrm{~b}$ illustrates the dislocation storage rate $\mathrm{d} \rho / \mathrm{d} \gamma$ as a function of plastic strain. The rate is constant during the second stage, and Figure $4 \mathrm{c}$ illustrates the saturated density rate as a function of $1 / \mathrm{bw}$, where they can be related through

$$
\frac{\mathrm{d} \rho}{\mathrm{d} \gamma}=2.17 \frac{1}{b w} \text {. }
$$

Relation (3) can be viewed as expressing a macroscopic storage rate, which is governed by a dislocation mean free path (Kocks, 1976). This result, i.e. a dislocation storage rate proportional to $1 / w$, means that the dislocation mean free path is mainly controlled by the material microstructure, and not by dislocations spacing as commonly observed in many materials. Here, it has been shown with the simulation (Vattre et al., 2010) that there is a noticeable decrease in the dislocation junction production rate after some straining. Hence the contribution of forest interaction to strain hardening is weak and the size-dependence of the flow stress cannot be correlated to the mean spacing between dislocations. Instead, as explained in the following, the plastic reponse is controlled here by the channel width. 


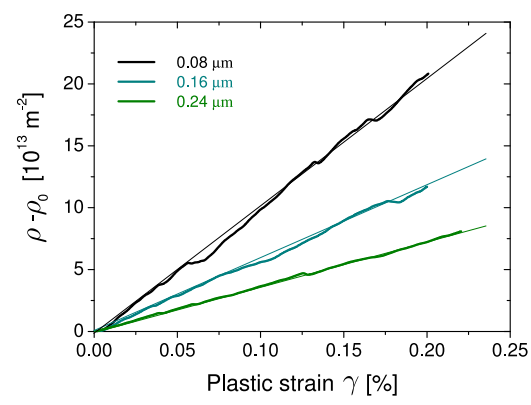

(a)

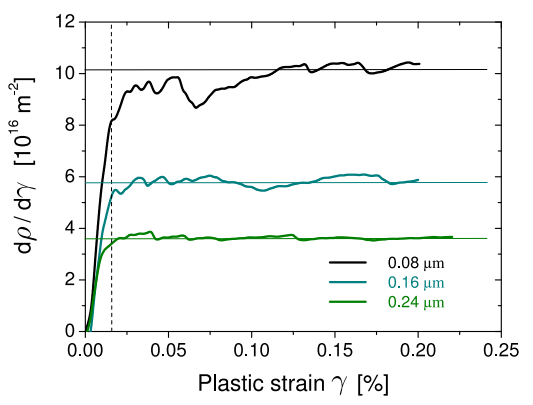

(b)

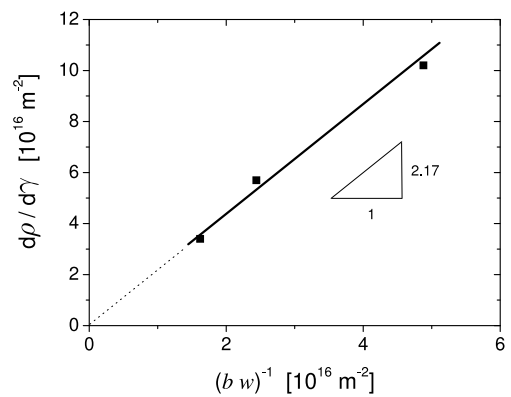

(c)

Figure 4. (a) Evolution of dislocation density with plastic strain corresponding to the stress-strain responses of Figure 3 a for all specimen sizes. (b) Rate of the total dislocation density with plastic strain. Horizontal lines represent the saturation rate during the second stage. (c) Saturation rate of the dislocation density evolution as a function of $1 / \mathrm{bw}$. The line represents a linear fit

\subsection{Distribution of dislocation density}

In order to quantify the local dislocation density variation, average densities were calculated over strips of $8 \mathrm{~nm}$ thickness running parallel to the interfaces. Two densities are considered: the total dislocation density, and the polarised dislocation density obtained by the Nye tensor $\boldsymbol{\alpha}$ (Nye, 1953). The latter gives the closure mismatch of a linear path traced on a three dimensional surface enclosing a volume $V$ containing an arbitrary dislocation microstructure. In the discrete sense, this tensor is computed by adding up the contributions of every dislocation segment according to

$$
\alpha_{i j}=\frac{1}{V} \sum_{k=1}^{N} l^{k} b_{i}^{k} t_{j}^{k},
$$

where $N$ is the number of segments in $V, l^{k}$ the length of segment $k, b^{k}$ its Burgers vector, and $t^{k}$ its unit line vector. Only a local polarised dislocation density produces a net, non-vanishing Nye's tensor. 


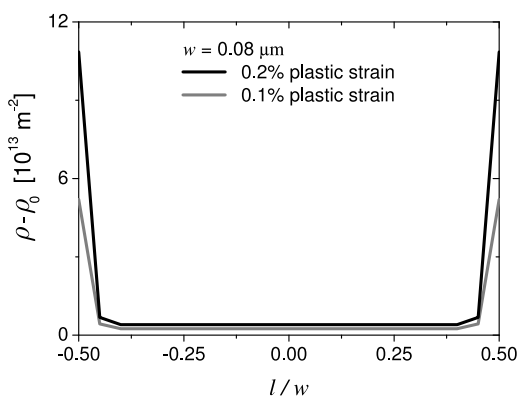

(a)

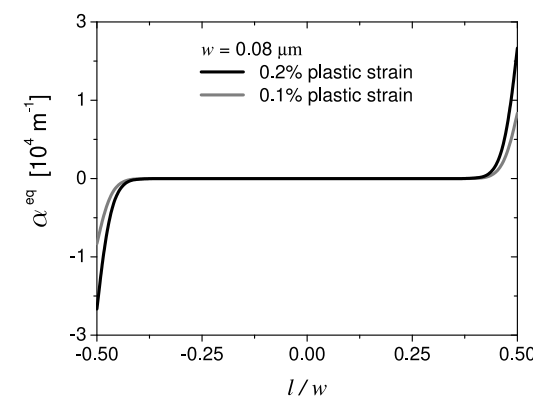

(b)

Figure 5. (a) Total dislocation density distributions across the channel at $0.1 \%$ and $0.2 \%$ plastic strain for $w=0.08 \mu \mathrm{m}$. (b) Averaged polarised dislocation density $\alpha^{\mathrm{eq}}$ calculated by Equation (4)

As shown in Figure 5a, the total dislocation density is higher at the interfaces than within the channel, and the density of these stored dislocations increases with ongoing deformation. No pile-ups are observed, contradicting 2D DD simulations. The corresponding averaged polarised dislocation density profiles $\alpha^{\mathrm{eq}}=\operatorname{sign}\left(\alpha_{11}\right) \sqrt{\alpha_{i j} \alpha_{i j}}$ are shown in Figure 5b. It shows that the dislocation densities located at the two interfaces are polarised. This strongly affects the local flow stress during [001] tensile loading because it induces internal stresses opposite to the applied stress, thereby reducing the dislocation mobility in the channels.

\section{Discussion}

As discussed in the previous section, when the Orowan critical shear stress is reached, the dislocations are sufficiently curved to glide into a channel. The channel width plays an important role in this threshold which, in the simulated results, is at about $0.01 \%$ plastic strain. The microplastic phase (i.e. from 0 to $0.01 \%$ ) corresponds to the bowout of preexisting dislocations in the channels between the interfaces. For larger channel widths, dislocations glide longer distances before being blocked by interfaces. The threshold stress depends on the channel width according to $1.02 \mu \mathrm{b} / \mathrm{w}$ (Equation 1). The theoretical value of this stress is also verified through collective effects and multi-slip conditions in 3D DD simulations.

When the critical Orowan stress is reached, a dislocation loop expands on a glide plane within the channel and deposits dislocation segments at the interfaces. These segments have a screw or $\pm 60^{\circ}$ mixed character, and give rise to a polarised dislocation density at the interfaces (Figure 5b). Their accumulation between the soft matrix and the hard precipitate generates internal stresses, and contributes strongly to the high work-hardening for $\langle 001\rangle$-oriented specimens. 


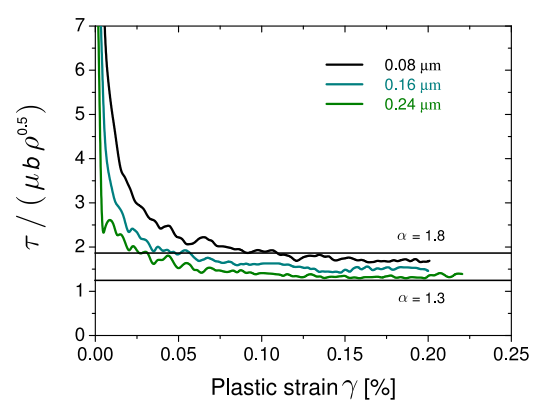

Figure 6. Proportionality factor $\alpha$ for Taylor hardening versus plastic strain, corresponding to Figures $3 a$ and $4 a$

The dislocation density increases almost linearly with plastic strain, and at the same $\gamma^{\prime}$ volume fraction the rate of increase is larger for smaller channel widths (Figure 4a). Moreover, the dislocation density rate $\mathrm{d} \rho / \mathrm{d} \gamma$ increases with decreasing channel width. In a consistent manner, strengthening is related to an increase of stresses inside the channels when the specimen size is reduced. This phenomena is associated with the emergence of a dislocation density polarised at the interfaces. Simulations of dislocation dynamics show that a network of interfacial dislocations is formed in 3D without pile-up and the interface dislocations accommodate the strain gradient between both phases.

In physical theories of crystal plasticity, the dislocation density is commonly used as the structural parameter for macroscopic descriptions of plastic flow, with the flow stress $\tau$ governed by Taylor-like hardening:

$$
\tau=\alpha \mu b \sqrt{\rho},
$$

where $\alpha$ is a constant in the range $0.3-0.5$ when plastic flow is controlled by forest interactions (Sevillano, 1993). Figure 6 shows the evolution of $\alpha$ with strain for the three simulated specimens. The stabilised values lie in the range $1.3-1.8$, far above the typical range of $0.3-0.5$. In other words, in these particular microstructures, the elementary mechanism restricting dislocation mobility is stronger than forest interactions. The $\gamma / \gamma^{\prime}$ interface with its polarised network of interface dislocations act as a strong barrier opposed to dislocation glide. Also, it must be noted that the evolution of $\rho$ is not the same as predicted by the classical storage-recovery model for dislocationdislocation interactions in bulk crystals. This is because here the storage rate is only function of the channel width (Equation 3).

\section{Conclusions}

The mechanical response of nickel-base single-crystal $\gamma / \gamma^{\prime}$ superalloys under [001] loading at $850^{\circ} \mathrm{C}$ has been simulated by the Discrete-Continuous Method 
(DCM). Within the DCM, plastic flow is directly simulated by a dislocation dynamics code, providing a physically justified manner for including dislocation glide, multiplication, annihilation and interactions with the $\gamma^{\prime}$ precipitates. Moreover, it contains an intrinsic length scale: the length of the Burgers vector. As at $850^{\circ} \mathrm{C}$ dislocations cannot penetrate into the precipitate, this work focused on the effect of the channel width on the macroscopic flow stress at constant $\gamma^{\prime}$ volume fraction. The main results are:

- The size effect is significant for both the flow stress and the work-hardening rate. The onset of the irreversible plastic regime below which dislocations cannot curve and glide within channels is inversely proportional to the channel width $w$.

- No pile-ups were observed in the simulations, so the mean free path is not reduced by a source-shortening mechanism. The dislocations are located at the interfaces, and the interfacial dislocation density increases by glide of mobile dislocations through the channel which deposit immobile segments at the interfaces.

- The proportionality factor $\alpha$ between the flow stress and the square root of the dislocation density is significantly higher $(1.3-1.8)$ in these microsctructures than in most single phased materials $(0.3-0.5)$. This is because the dislocation density is not distributed randomly but into a polarised interfacial network, thereby creating a stress field in the channels opposing dislocation motion.

These findings raise fundamental questions regarding the formulation of extended plasticity theories for modelling work hardening, which should include the long-range internal stress generated by the polarised dislocation density.

\section{References}

Acharya A., Bassani J. L., "Incompatibility and crystal plasticity”, J. Mech. Phys. Sol., vol. 48, p. $1565-1595,2000$.

Busso E. P., Meissonnier F. T., O’Dowd N. P., “ Gradient-dependent deformation of two-phase single crystals”, J. Mech. Phys. Sol., vol. 48, p. 2333-2361, 2000.

Carry C., Strudel J. L., “ Apparent and effective creep parameters in single crystals of a nickel base superalloy - I. Incubation period”, Acta Met., vol. 25, p. 767-777, 1977.

Cleveringa H. H. M., Van Der Giessen E., " Discrete dislocation simulations and size dependent hardening in single slip", J. de Physique, vol. 8, p. 83-92, 1998.

Cleveringa H. H. M., Van Der Giessen E., Needleman A., " Comparison of discrete dislocation and continuum plasticity predictions for a composite material", Acta Mat., vol. 45, p. 31633179, 1997.

Devincre B., Roos A., Groh S., “ Boundary problems in DD simulations. In: Thermodynamics, Microstructures and Plasticity, A. Finel et al., Nato Sciences Series II: Mathematics, Physics and Chemistry", , vol. 108, p. 275, 2003.

Duhl D. N., “ Directionally solidified superalloys.”, in C. Sims, N. Stoloff, W. C. Hagel (eds), Superalloys II: High Temperature Materials for Aerospace and Industrial Power, J. Wiley \& Sons, New York, p. 189-214, 1987. 
Fivel M., Canova G. R., “ Developing rigorous boundary conditions to discrete dislocation dynamics”, Mod. Sim. Mat. Sc. Eng., vol. 7, p. 753-768, 1999.

Forest S., Barbe F., Cailletaud G., " Cosserat modelling of size effects in the mechanical behavior of plocrystal and multi-phase materials", Int. J. Sol. Struct., vol. 37, p. 7105-7126, 2000.

Groh S., Devincre B., Kubin L., Roos A., Feyel F., Chaboche J.-L., " Size effects in metal matrix composites", Materials Science and Engineering A, vol. 400-401, p. 279-282, 2005.

Gurtin M. E., " On the plasticity of single crystals: free energy, microforces, plastic-strain gradients”, J. Mech. Phys. Sol., vol. 48, p. 898-1036, 2000.

Kocks U. E., “ Laws for work-hardening and low-temperature creep”, Journal of Engineering Materials and Technology, vol. 98, p. 16-85, 1976.

Lemarchand C., Devincre B., Kubin L. P., " Homogenization method for a discrete-continuum simulation of dislocation dynamics”, J. Mech. Phys. Sol., vol. 49, p. 1969-1982, 2001.

Madec R., Devincre B., Kubin L. P., " On the use of periodic boundary conditions in dislocation dynamics simulations", in Y. Shibutani, H. Kitagawa (eds), IUTAM Symposium on Mesoscopic Dynamics in Fracture Process and Strength of Materials, vol. 115, Kluwer, Dordrecht, p. 35-44, 2003.

Mura T., Micromechanics of defects in solids, Kluwer Academic Publishers, 1987.

Nouailhas D., Lhuillier S., “ On the micro-macro modelling of $\gamma / \gamma^{\prime}$ single crystal behavior”, Comp. Mat. Sc., vol. 9, p. 177-187, 1997.

Nye J. F., " Some geometrical relations in dislocated crystals", Acta Met., vol. 1, p. 153-162, 1953.

Pollock T. M., Argon A. R., “ Creep resistance of CMSX-3 nickel base superalloy single crystals”, Acta Met., vol. 40, p. 1-30, 1992.

Sevillano G., "Low stress and work hardening. In: Cahn, R.W. Haasen, P. Kramer, E. Mughrabi (Eds), Materials Science and Technology”, , vol. 6, p. 19-88, 1993.

Shu J. Y., Fleck N. A., Van Der Giessen E., Needleman A., “ Boundary layers in constrained plastic flow: comparison of nonlocal and discrete dislocation plasticity", J. Mech. Phys. Sol., vol. 49, p. 1361-1395, 2001.

Van der Giessen E., Needleman A., “ Discrete Dislocation Plasticity - A Simple Planar Model”, Mod. Sim. Mat. Sc. Eng., vol. 3, p. 689-735, 1995.

Vattré A., Devincre B., Roos A., " Dislocation Dynamics simulations of precipitation hardening in Ni-based superalloys with high $\gamma^{\prime}$ volume fraction", Intermetallics, vol. 17, p. 988-994, 2009.

Vattré A., Devincre B., Roos A., " Orientation dependence of plastic deformation in nickel-base single crystal superalloys: a Discrete-Continuous Model simulation”, Acta Mat., 2010.

Veyssière P., Saada G., " Microscopy and plasticity of the $\mathrm{L}_{2} \gamma^{\prime}$ phase, in 'Dislocations in Solids"”, , vol. 10, p. 253, 1997.

Westbrook J. H., Intermetallic Compounds, John Wiley, 1967.

Zbib H. M., De la Rubia T., “ A Multiscale Model of Plasticity”, Int. J. Plast., vol. 18, p. 1133$1163,2002$. 